\title{
Chemical and Electrochemical Synthesis of Highly Conductive and Processable PolyProDOP-alkyl Derivatives
}

\author{
Younkyung Cho, Myoungho Pyo*, and Kyukwan Zong ${ }^{\dagger * *}$ \\ Department of Printed Electronics, Sunchon National University, Chonnam 540-742, Korea \\ ${ }^{\dagger}$ Institute of Science Education, Division of Science Education, Chonbuk National University, \\ Chonbuk 561-756, Korea
}

(Received January 29, 2010 : Accepted February 16, 2010)

\begin{abstract}
New monomers, possessing various alkyl substituents on propylene dioxypyrrole, were synthesized. The monomers could be easily polymerized to produce highly conductive and soluble polymers. The corresponding polymers showed excellent solubility, retaining electrochemical and optical properties of their parent polymer [poly(propylene dioxypyrrole)]. The conductivities of chemically prepared polymers were quite high in a range of 20 and $60 \mathrm{Scm}^{-1}$. Solubility of the polymer in a common organic solvent was as high as no polymer is deposited on an electrode. The redox potentials of the electrochemically prepared polymers revealed quite stable electroactivity during repeated redox switching up to 500 times. The optoelectrochemistry studies also showed distinct color changes of the polymers upon changing the doping state, indicating strong absorption peaks at $400 \sim 600 \mathrm{~nm}$ in reduced states and complete bleaching in fully oxidized states.
\end{abstract}

Keywords : Conducting polymer, ProDOP, Soluble polymer, Conductivity

\section{Introduction}

There has been enormous interests in the area of electrically conducting polymers for decades since they have a great potential for practical applications to a wide variety of areas. ${ }^{1,3)}$ Among them, polypyrrole has been one of the most widely studied conducting polymers due to its low oxidation potential and relatively high conductivity. ${ }^{4)}$ However, poor processability and inferior long term stability of polypyrrole hampered the large scale applications in industrial fields.

Recently, Reynolds group reported a series of studies in that the polymers (PXDOPs) from 3,4-alkylenedioxypyrroles (XDOPs) were highly conductive and excellently stable in a high number of redox switches with little degradation in charge response. ${ }^{5-8)}$ By analogy with 3,4-ethylendioxythiophene (EDOT), ${ }^{9)}$ an appending dioxyalkylene group across the 3- and 4- positions of the pyrrole leads to the polymer with vastly improved properties compared to the parent. With excellent physical properties of PXDOPs, they envisioned that

*E-mail: mho@sunchon.ac.kr, kzong@jbnu.ac.kr the polymers could be tailored to be processable by appending a alkyl chain to the alkylene bridge of 3,4propylenedioxypyrrole (ProDOP).

Here, we wish to report new polymers which are highly conductive and processable. Poly(3,4-propylenedioxy)pyrryl alkyl derivatives, that are both chemically and electrochemically polymerized from corresponding monomers, were examined. Conductivity, solubility, and (opto)electrochemical properties of octyl- (PProDOPOct), ethylhexyl- (PProDOP-EthHex), and dodecyl(PProDOP-Dod) deriveratives were compared.

\section{Experimental}

\subsection{Gneral Considerations}

${ }^{1} \mathrm{H}$ NMR $(300 \mathrm{MHz})$ and ${ }^{13} \mathrm{C}$ NMR $(75 \mathrm{MHz})$ spectra were recorded on a Varian VXR-300. Elemental analyses were carried out by Robertson Microlit Laboratories, Inc. Gel Permeation Chromatography (GPC) was performed using a Waters Associations liquid chromatography U6K equipped with tandem ABI Spectraflow 757 UV absorbance detector and a Perkin-Elmer LC-25 RI detector. The GPC solvent delivery system was config- 
ured for elution with chloroform through an Ultrastyragel linear mixed bed column at a rate of $1.0 \mathrm{~mL} / \mathrm{min}$. Retention times were calibrated against eight narrow (PDI < 1.07) polystyrene standards (Scientific Products, Inc.) with $\mathrm{Mp}=1.35 \times 10^{3}-8.6 \times 10^{5} \mathrm{~g} / \mathrm{mol}$. Spectroscopic data were recorded on a Varian Cary 5E UV-VIS-NIR spectrophotometer. Electrochemical synthesis and characterization of polymers were performed with EC Epsilon. The working electrodes were Pt or ITO (Delta Tech.), the potential of which were controlled vs. $\mathrm{Ag} / \mathrm{AgCl}$ reference electrode. Conductivity was measured by 4 point probes (Dasol eng, Korea).

\subsection{Monomer and Polymer Synthesis}

All monomer synthesis is described in previous articles (Fig. 1) ${ }^{5-8)}$ For chemical synthesis of polymer, into a $100 \mathrm{~mL}$ three necked flasked equiped with a magnetic stirring bar, reflux condenser, and dropping funnel was placed $6 \mathrm{mmol}$ of a monomer in $30 \mathrm{~mL}$ of methanol. The solution of an oxidant in methanol was charged into the dropping funnel and added to the solution containing a monomer with vigorous stirring at $0^{\circ} \mathrm{C}$. A Black precipitate was filtered through a glass frit funnel and washed with methanol several times. A collected polymer was suspended into chloroform with hydrazine and the mixture was stirred at reflux for overnight. The reaction mixture was cooled to room temperature and re-precipitated to remove low molecular weight molecules by adding methanol. The polymer was filtered, washed with methanol several times, and dried under vacuum to give the desired product. Elemental analysis of polymers (doped); PolyProDOP-Oct, found: C, 65.37; H, 8.71; N, 4.99; PolyProDOP-EthHex, found: C, 65.12; H, 8.07; N, 4.77;
PolyProDOP-Dod, found: C, 70.09; H, 9.72; N, 4.16.

\section{Results and Discussion}

\subsection{Monomer synthesis and chemical polymer-} ization

Three monomers were synthesized according to the method that has been developed previously ${ }^{6-8)}$ (see Fig. 1) and they were chemically polymerized by the method developed by Machida and coworkers. ${ }^{10)}$ In the chemical polymerization, anhydrous $\mathrm{FeCl}_{3}$ turned out most effective in polymerizing our monomers than other oxidants. The reactions gave rise to relatively higher molecular weight polymers in good yields (Fig. 3). GPC analyses of the polymers, performed by comparison with polystyrene standards using UV-VIS detector, showed that they have relatively a broad range of molecular distributions having high polydispersities (PDI 7). A typical molecular weight (Mw) of PProDOP-Oct ranged around 28,000 and number-average molecular weight $(\mathrm{Mn})$ around 4,000 indicative of $10 \sim 16$ repeating units in the polymer chain.

\subsection{Solubility and conductivity of polymers}

Under the similar condition, the polymerization of these monomers with $\mathrm{CuCl}_{2}$ resulted in low molecular weight polymers or oligomers in low yields. In varying solvents, the use of methanol in polymerizing these monomers turned out to be a better choice than chloroform in terms of yield, physical property, and conductivity of the resulting polymers. Chemically synthesized polymers were reduced to neutral forms by treating with hydrazine or ammonia by refluxing for a day. As the polymers were reduced to be neutral, the color of the solution was

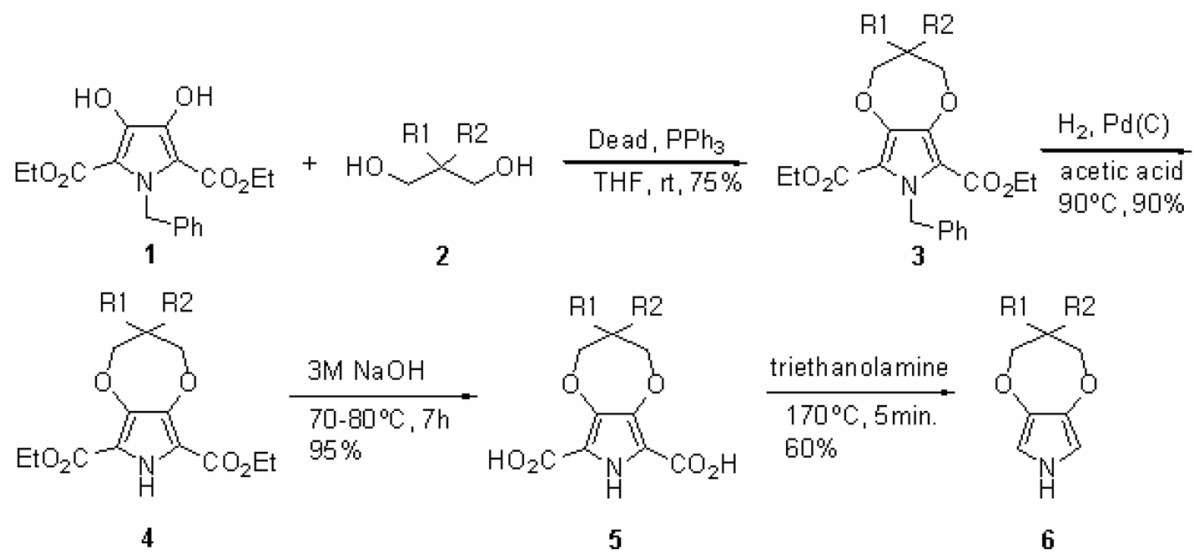

Fig. 1. Synthetic scheme of monomers. 


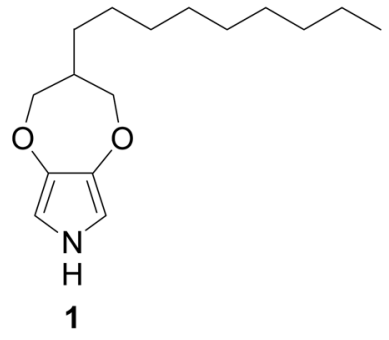

ProDOP-Oct<smiles>CCCCC(CC)CC1COc2c[nH]cc2OC1</smiles>

ProDOP-EthHex

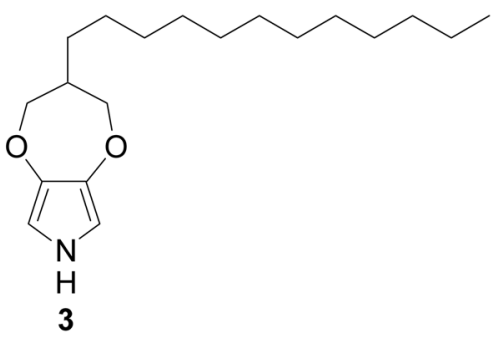

ProDOP-Dod

Fig. 2. Structure of monomers.

changed black to dark pink and the polymers were dissolved completely. After purified by reprecipitation, neutral polymers obtained were soluble in chloroform, dichloromethane, dimethylformamide but partially soluble in tetrahydrofurane. Neutral polymers were characterized by proton NMR spectroscopy. Generally, the spectra of neutral polymers gave perfectly matched peaks with those of monomer spectra, but the peaks of the polymers were broader as can be seen in those of other types of soluble polymers. Broadness of the peaks possibly comes from incomplete reduction of the polymers that were initially synthesized in a doped form. However, we have not succeeded in obtaining completely reduced states of our polymers in spite of many attempts. This may attribute to highly electron-rich property of our polymers and difficulty for these polymers in maintaining their neutral states under normal conditions due to high sensitivity to air. The attempts of these polymers to protect from air brought little improvement.

The conductivity of all polymers was measured using a four probe method. The polymer prepared by using a simple filtration device. A polymer dispersed solution that was initially prepared from polymerization of a monomer using anhydrous $\mathrm{FeCl}_{3}$ in methanol was poured into a fritted funnel and applied to vacuum to make a uniformed film. Since ProDOP has a flexible alkyl chain appended to the propylenedioxy ring as shown in Fig. 2, the resulting polymer afforded a dense, glossy, and highly flexible film. However, the films from PProDOP-Oct and PProDOP-Dod gave relatively less dense and flexible ones. The film thickness prepared was within $80-90 \mu \mathrm{m}$. The films prepared from PPro-<smiles>[X]C1COc2c[nH]cc2OC1</smiles><smiles>CC(C)(C)O[Na]</smiles>

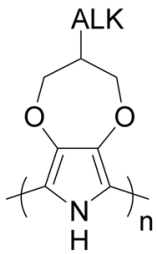<smiles>CCCCCCCCCC1COc2c(C(C)(C)C)[nH]c(C(F)(F)F)c2OC1</smiles><smiles>CCCCC(CC)CC1COc2c(C(C)(C)C)[nH]c(C(F)(F)F)c2OC1</smiles>

PProDOP-Oct

PProDOP-EthHex

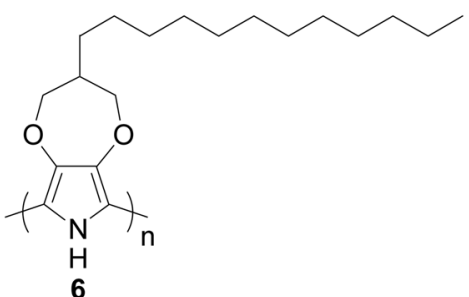

PProDOP-Dod

Fig. 3. Chemical polymerization and structure of resulting polymers. 
Table 1. Conditions of Chemical Polymerization of ProDOP-Alkyls and the Yields and Conductivity of the Polymers

\begin{tabular}{lccccccc}
\hline Monomers & Oxidants & $\begin{array}{c}\text { Equivalent of Oxi- } \\
\text { dant }\end{array}$ & Solvents & $\begin{array}{c}\text { Reaction Temp. } \\
\left({ }^{\circ} \mathrm{C}\right)\end{array}$ & $\begin{array}{c}\text { Reaction Time } \\
(\mathrm{h})\end{array}$ & $\begin{array}{c}\text { Conductivity } \\
\left(\mathrm{Scm}^{-1}\right)\end{array}$ & $\begin{array}{c}\text { Yields } \\
(\%)\end{array}$ \\
\hline ProDOP-Oct & $\mathrm{CuCl}_{2}$ & 2.33 & $\mathrm{MeOH}$ & 25 & 12 & - & $40-60$ \\
ProDOP-Oct & $\mathrm{FeCl}_{3}$ & 3.00 & $\mathrm{CHCl}$ & 25 & 12 & - & $30-45$ \\
ProDOP-Oct & $\mathrm{FeCl}_{3}$ & 2.33 & $\mathrm{MeOH}$ & 0 & 3 & 24 & $85-90$ \\
ProDOP-EthHex & $\mathrm{FeCl}_{3}$ & 2.33 & $\mathrm{MeOH}$ & 0 & 3 & 25 & $89-95$ \\
ProCOP-Dod & $\mathrm{FeCl}_{3}$ & 2.33 & $\mathrm{MeOH}$ & 0 & 3 & 55 & $85-90$ \\
\hline
\end{tabular}

DOP-EthHex, PProDOPOct, and PProDOP-Dod turned out highly conductive, revealing 55, 24, and $25 \mathrm{Scm}^{-1}$ respectively. The reason of slightly less conductivity for PProDOPOct and PProDOP-Dod may attribute to the film quality of those polymers (i.e.; less dense films). Typically, PProDOP-EthHex film turned out highly stable and did not lose conductivity even after it had been exposed to open area for a month. The conductivities along with the polymerization conditions were summarized in Table 1.

The polymers were further studied by doping the solution containing the neutral polymers with $0.01 \mathrm{M} \mathrm{SbCl}_{5}$ in $\mathrm{CH}_{2} \mathrm{Cl}_{2}$. From the UV-VIS spectra, it was found that the neutral polymer did not give rise to any significant $\pi-\pi *$ absorption in a UV-VIS region in spite of various attempts, indicating that the polymer was not fully reduced to neutral state. As revealed in NMR spectroscopy, it is believed that fully reduced state by chemical treatment seemed to be very difficult to achieve under normal condition.

\subsection{Electrochemical studies of polymers}

The electropolymerization of ProDOP derivatives can be done easily in a conventional potential range and result in the fast growth as shown in Fig. 4 during PProDOP-Oct synthesis. The monomer oxidation potential and redox potential of the polymer are quite similar to the values of ProDOP, indicating that the alkyl substituents simply enhance the polymer solubility without affecting the electronic structure. It should be mentioned that the films are not formed on the electrode in organic solvents (acetonitrile or $\mathrm{CH}_{2} \mathrm{Cl}_{2}$ ) due to high solubility of polymers. Therefore, the electropolymerization and subsequent voltammetric studies were performed in a mixed solvent $\left(\mathrm{H}_{2} \mathrm{O} /\right.$ acetonitrile $\left.=1 / 1\right)$.

The cyclic voltammograms (CV) of 3 polymers seem to be quite similar. Fig. 5 shows CV of PProDOP-Oct as an example. Anodic and cathodic peaks are distinct and the peak heights proportionally increase as the

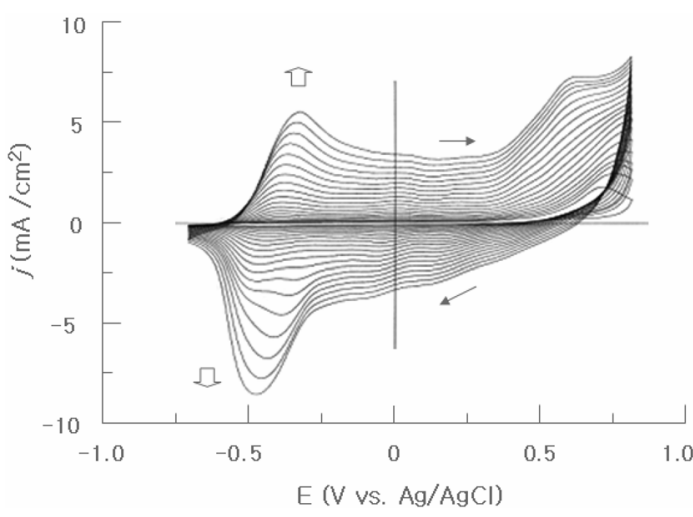

Fig. 4. Cyclic voltammograms during electrochemical synthesis of PProDOP-Oct on $\mathrm{Au}$ in acetonitrile/ $\mathrm{H}_{2} \mathrm{O}$ cosolvent (1/1) of $50 \mathrm{mM} \mathrm{LiClO}_{4} . v=50 \mathrm{mVs}^{-1}$.

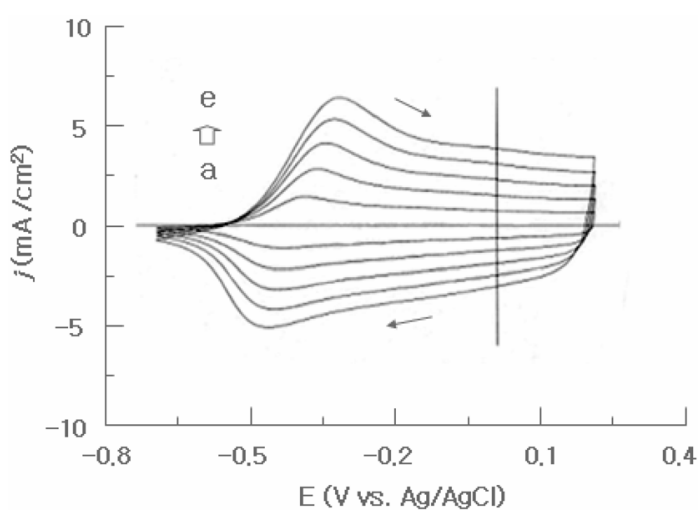

Fig. 5. Cyclic voltammograms of PProDOP-Oct in acetonitrile/ $\mathrm{H}_{2} \mathrm{O}$ cosolvent (1/1) of $50 \mathrm{mM} \mathrm{LiClO}_{4}, v=$ (a) 20, (b) 40, (c) 60, (d) 80 , and (e) $100 \mathrm{mVs}^{-1}$.

increase of scan rate. These polymers are quite stable under repeated redox switching. PProDOP-Oct was subjected to potential cycling in between -0.7 and $+0.2 \mathrm{~V}$ at $50 \mathrm{mV} / \mathrm{s}$. Fig. 6 compares the initial and $500^{\text {th }} \mathrm{CV}$ of PProDop-Oct. It is obvious that the electroactivity of the polymer is retained although the electroactivity is decreased by $30 \%$. This electrochemical stability 


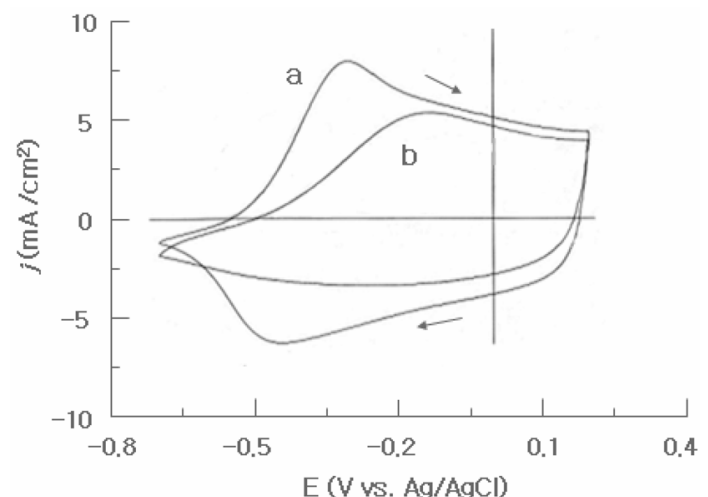

Fig. 6. Cyclic voltammograms of PProDOP-Oct in acetonitrile/ $\mathrm{H}_{2} \mathrm{O}$ cosolvent (1/1) of $100 \mathrm{mM} \mathrm{LiClO}_{4}$. (a) initial voltammogram and (b) $500^{\text {th }}$ voltammogram, showing stability of PProDOP-Oct during extensive redox switching.

coincides with environmental stability of the chemically polymerized polymers described above.

We also compared optical absorption of the polymers during potential application. Fig. 7 shows UV-Vis absorption spectra of PProDOP-Oct (top) and PProDOP-Dod (bottom). The potentials were controlled for the polymers to be in fully oxidized and reduced states. When in a fully reduced state, the polymer strongly absorbs lights between 400 and $600 \mathrm{~nm}$, demonstrating distinct vibronic transitions. As the increase of the potential, these characteristic behaviors are weakened and finally disappear. The absorption maximum shifts to the NIR region due to inner band formation.

\section{Conclusions}

New monomers (ProDOP-EthHex, ProDOP-Oct, and ProDOP-Dod) were synthesized. The corresponding polymers showed excellent solubility while maintaining electrochemical and optical properties of their parent polymer (PProDOP). The conductivities of chemically prepared polymers were quite high in a range of 20 and $60 \mathrm{Scm}^{-1}$, which are maintained after one month in air. Solubility of the polymer in a common organic solvent was as high as no polymer is deposited on an electrode. The coexistence of $\mathrm{H}_{2} \mathrm{O}$ is required to make a smooth film. The redox potentials of the electrochemically prepared polymers were similar to the one of PProDOP, revealing quite stable electroactivity after repeated redox switching up to 500 times. The optoelectrochemistry studies showed distinct color changes of the polymers during redox, indicating strong absorption peaks
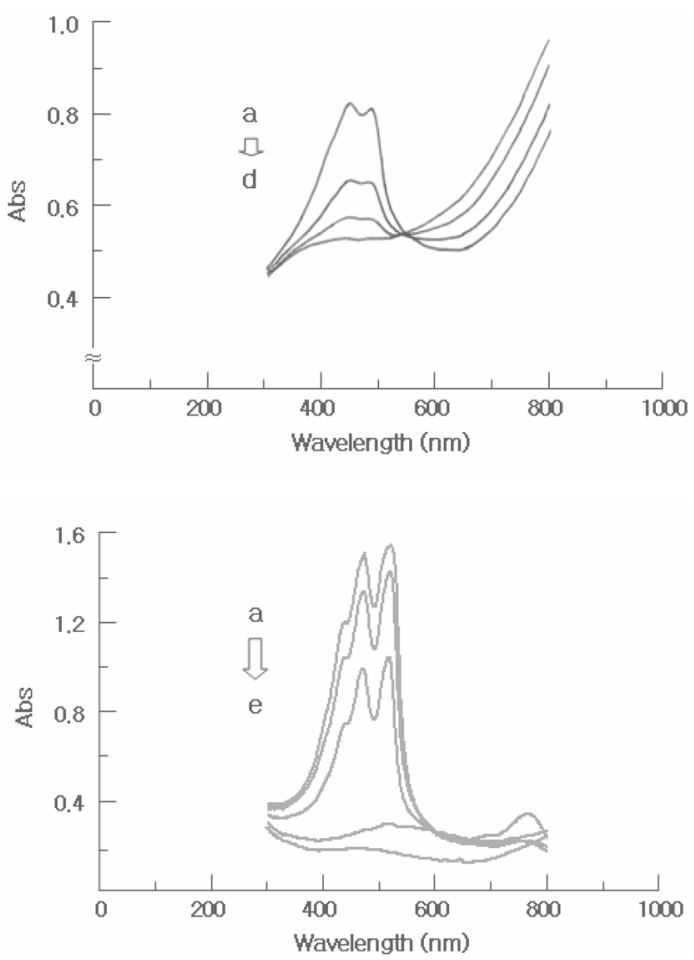

Fig. 7. Absorption spectra of PProDOP-Oct (top) and PProDOP-Dod (bottom) in $50 \mathrm{mM} \mathrm{LiClO}_{4}$ at the potential of (a) -0.6 , (b) -0.4 , (c) -0.2 , (d) +0.4 , and (e) $+0.4 \mathrm{~V}$.

at $400-600 \mathrm{~nm}$ in reduced states and complete bleaching in fully oxidized states.

\section{Acknowledgment}

This work was supported by the Korea Research Foundation Grant funded by the Korean Government (KRF-2006-521-C00080).

\section{References}

1. N. Toshima and S. Hara, 'Direct synthesis of conducting polymers from simple monomers' Prog. Polym. Sci., 20, 155 (1995).

2. Handbook of Conducting Polymers, $2^{\text {nd }}$ ed. (Eds: T. A. Skoteheim, R. L. Elsenbaumer, and J. R. Reynolds), Dekker, New York, (1998).

3. M. Salmon, K. Kanazawa, A. F. Diaz, and M. Krounbi, 'A chemical route to pyrrole polymer films' J. Polym. Sci., Polym. Lett., 22, 187 (1982).

4. J. Hwang and M. Pyo, 'pH-induced mass and volume changes of perchlorate-doped polypyrrole' Synth. Met., 157, 155, (2007). 
5. M. Pomerantz, J. J. Tseng, H. Zhu, S. J. Sproull, J. R. Reynolds, R. Uitz, H. J. Arnott, and M. I. Haider, 'Processable polymers and copolymers of 3-alkylthiophenes and their blends' Synth. Met., 41-43, 825 (1991).

6. C. A. Thomas, K. Zong, P. Schottland, and J. R. Reynolds, 'Poly(3,4-alkylenedioxypyrrole)s as highly stable aqueouscompatible conducting polymers with biomedical implications' Adv. Mater., 12, 222 (2000).

7. P. Schottland, K. Zong, C. L. Gaupp, B. C. Thompson, C. A. Thomas, I. Giurgiu, R. Hickman, K. A. Abboud, and J. R. Reynolds, 'Poly(3,4-alkylenedioxypyrrole)s: highly stable electronically conducting and electrochromic polymers' Macromolecules, 33, 7051 (2000).

8. R. M. Walczak, J. Jung, J. S. Cowart, and J. R. Reynolds,
'3,4-Alkylenedioxypyrrole-based conjugated polymers with finely tuned electronic and optical properties via a flexible and efficient $N$-functionalization method' Macromolecules, 40, 7777 (2007).

9. W. J. Doherty III, R. J. Wysocki, N. R. Aemstrong, and S. S. Saaverdra, 'Electrochemical copolymerization and spectroelectrochemical characterization of 3,4-ethylenedioxythiophene and 3,4-ethylenedioxythiophenemethanol copolymers on indiumtin oxide' Macromolecules, 39, 4418 (2006).

10. S. Machida, S. Miyata, and A. Techagumpuch, 'Chemical synthesis of highly electrically conductive polypyrrole' Synth. Met., 31, 311 (1989). 